Lei Wang, Yu-Ran Wu, Shu-Ting Ren, Long Yin, Xiu-Jian Liu, Feng-Chang Cheng, Wei-Wei Liu*, Da-Hua Shi, Zhi-Ling Cao and Hui-Min Sun

\title{
Synthesis and bioactivity of novel C2-glycosyl oxadiazole derivatives as acetylcholinesterase inhibitors
}

https://doi.org/10.1515/hc-2018-0166

Received September 4, 2018; accepted October 4, 2018; previously published online November 13, 2018

\begin{abstract}
A series of glycosyl-substituted 1,3,4-oxadiazoles were synthesized by cyclization of glycosyl-acylthiosemicarbazides via a base-catalyzed reaction. The starting glycosyl-acylthiosemicarbazide derivatives were obtained by the reaction of glycosyl isothiocyanate with various hydrazides. The acetylcholinesterase (AChE) inhibitory activities of the products were tested by Ellman's method. The most active compounds were subsequently evaluated for the $50 \%$ inhibitory concentration (IC $\left.{ }_{50}\right)$ values. $N$-(1,3,4,6-tetra- $O$-benzyl-2-deoxy- $\beta$-D-glucopyranosyl)-5-(4fluorophenyl)-1,3,4-oxadiazole-2-amine (6i) possesses the best AChE -inhibition activity with an $\mathrm{IC}_{50}$ of $1.61 \pm 0.34 \mu \mathrm{M}$.
\end{abstract}

Keywords: Acetylcholinesterase inhibitors; glycosyl 1,3,4-oxadiazoles; synthesis.

\section{Introduction}

Carbohydrates have long interested chemists and biochemists as a large natural resource [1-4]. As an energy source and an element of many metabolic processes, sugars are present in every part of the human body [5].

\footnotetext{
*Corresponding author: Wei-Wei Liu, College of Pharmaceutical Sciences, Huaihai Institute of Technology, Lianyungang 222005 , P.R. China; Jiangsu Institute of Marine Resources, Lianyungang 222005, P.R. China; Jiangsu Key Laboratory of Marine Pharmaceutical Compound Screening, Huaihai Institute of Technology, Lianyungang 222005, P.R. China; and Co-Innovation Center of Jiangsu Marine Bio-industry Technology, Lianyungang 222005, P.R. China, e-mail: liuweiwei255@163.com Lei Wang, Yu-Ran Wu, Shu-Ting Ren, Long Yin, Xiu-Jian Liu and Hui-Min Sun: College of Pharmaceutical Sciences, Huaihai Institute of Technology, Lianyungang 222005, P.R. China

Feng-Chang Cheng: China University of Mining and Technology, Xuzhou 221116, P.R. China

Da-Hua Shi and Zhi-Ling Cao: College of Pharmaceutical Sciences, Huaihai Institute of Technology, Lianyungang 222005, P.R. China; and Jiangsu Institute of Marine Resources, Lianyungang 222005, P.R. China
}

D-glucosamine is a naturally occurring amino sugar $[6,7]$, one of the most abundant monosaccharides, and has been widely used in the prevention and/or treatment of rheumatoid arthritis and osteoarthritis [8, 9]. Furthermore, D-glucosamine exhibits a broad variety of bioactivities such as anti-inflammatory [10], anti-cancer [11] and antibacterial [12] properties and it suppresses tumor growth [13]. Modified carbohydrates provide access to potential mimetics of naturally occurring amino sugars and represent targets for the development of anti-oxidant [14], antiacetylcholinesterase (AChE) [15], anti-proliferative [16] and other active agents [17-20].

The emergence of heterocyclic compounds with novel structures may promote discovery of new drugs and treatment of stubborn diseases [21-24]. Recently, the oxadiazole chemistry has been developed extensively. 1,3,4-Oxadiazoles structurally resemble amides and esters [25], and some compounds show similar pharmacokinetic properties [26-31]. Moreover, recent studies have shown that a number of compounds containing the 1,3,4-oxadiazole skeleton act as monoamine oxidase inhibitors for the treatment of Alzheimer's disease (AD) [32-34]. AD is a chronic, neurodegenerative disorder that causes an irreversible dementia in elderly people [35].

Researchers have been interested in molecular hybridbased approaches to find new compounds of potential biological activities [36, 37]. Based on the aforementioned information, herein we report the design and synthesis of novel D-glucosamine/1,3,4-oxadiazole hybrids with the oxadiazole ring offering an important pharmacophore to discover new potent $\mathrm{AChE}$ inhibitors. The synthesized derivatives were evaluated by Ellman's method for AChE inhibitors and to explore the influence of D-glucosamine against AChE inhibition.

\section{Results and discussion}

\section{Chemistry}

In the development of C2-glycosyl oxadiazoles, glycosyl isothiocyanate 3 was the critical intermediate compound. 


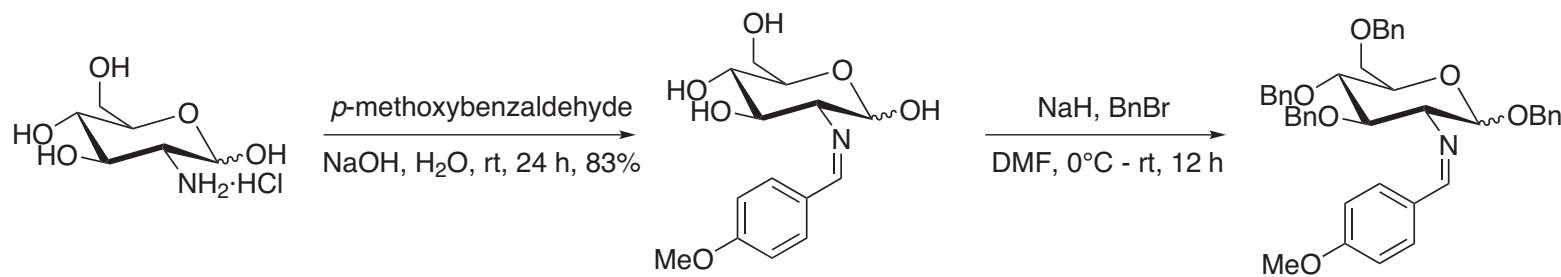

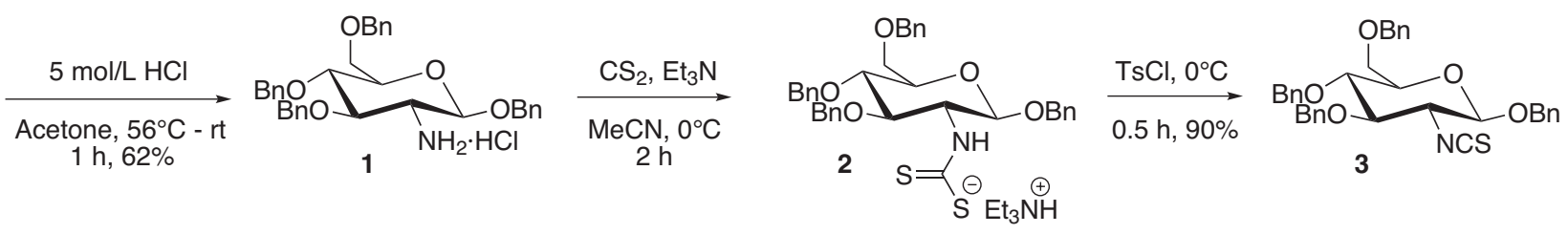

Scheme 1

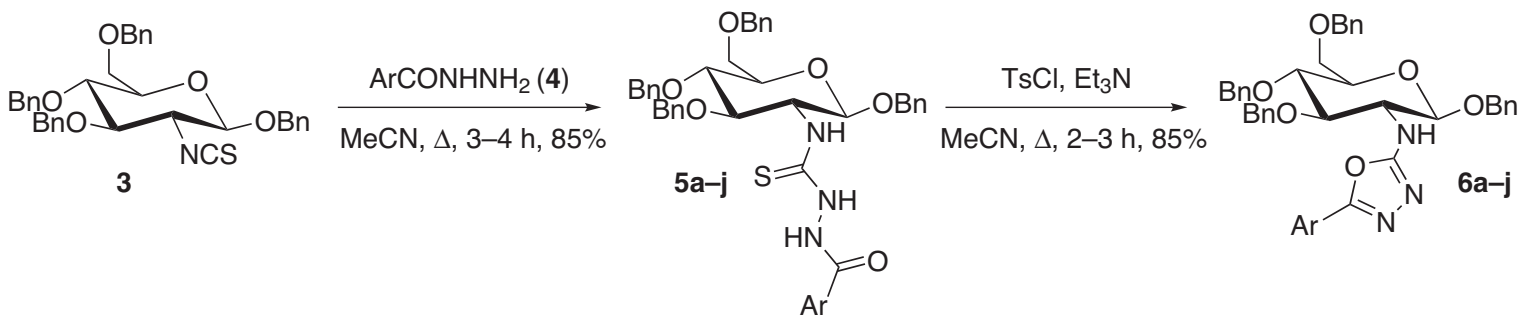

$$
\begin{aligned}
& \text { a: } \mathrm{Ar}=\mathrm{C}_{6} \mathrm{H}_{5} \\
& \text { b: } \mathrm{Ar}=4-\mathrm{CH}_{3}-\mathrm{C}_{6} \mathrm{H}_{4} \\
& \text { c: } \mathrm{Ar}=2 \text {-thienyl } \\
& \text { d: } \mathrm{Ar}=4-\text { pyridyl } \\
& \text { e: } \mathrm{Ar}=3-\mathrm{OCH}_{3}-\mathrm{C}_{6} \mathrm{H}_{4}
\end{aligned}
$$

f: $\mathrm{Ar}=2-\mathrm{Cl}-\mathrm{C}_{6} \mathrm{H}_{4}$

g: $\mathrm{Ar}=4-\mathrm{OH}-\mathrm{C}_{6} \mathrm{H}_{4}$

h: $\mathrm{Ar}=4-\mathrm{I}-\mathrm{C}_{6} \mathrm{H}_{4}$

i: $\mathrm{Ar}=4-\mathrm{F}-\mathrm{C}_{6} \mathrm{H}_{4}$

j: $\mathrm{Ar}=4-(\mathrm{N}, \mathrm{N}-\mathrm{di}-\mathrm{Me})-\mathrm{C}_{6} \mathrm{H}_{4}$

Scheme 2

Table 1 In vitro inhibitory activities of glycosyl oxadiazoles against AChE.

\begin{tabular}{lrr}
\hline Compound & ${\text { Inhibition }(\%)^{\mathrm{a}}}$ & $\mathrm{IC}_{50}(\mu \mathrm{M})$ \\
\hline $\mathbf{1}$ & 46.2 & - \\
$\mathbf{6 a}$ & 47.7 & - \\
$\mathbf{6 b}$ & 59.4 & - \\
6c & 92.1 & $2.6 \pm 0.5$ \\
6d & 90.2 & $4.2 \pm 0.2$ \\
6e & 55.1 & - \\
6f & 91.3 & $3.1 \pm 0.4$ \\
6g & 68.3 & - \\
6h & 83.3 & $11.6 \pm 0.8$ \\
6i & 96.8 & $1.6 \pm 0.3$ \\
6j & 91.1 & $2.7 \pm 0.5$ \\
$\mathbf{m}^{\mathrm{b}}$ & 17.3 & - \\
$\mathrm{n}^{\mathrm{c}}$ & 14.5 & - \\
Tacrine & & $0.269 \pm 0.004$ \\
Galantamine & & $2.67 \pm 0.15$ \\
\hline
\end{tabular}

aThe inhibition activities of the compounds at the concentration of $50 \mu \mathrm{g} / \mathrm{mL}$. ${ }^{b} \mathrm{~m}$ stands for 5-(4-(N,N-di-Me)- $\left.\mathrm{C}_{6} \mathrm{H}_{4}\right)-1,3,4$-oxadiazole-2amine. ${ }^{\mathrm{n}} \mathrm{n}$ stands for $\mathrm{D}$-glucosamine hydrochloride.

Our strategy began with an attempted synthesis of this starting material without protection of hydroxyl groups at D-glucosamine hydrochloride but, to our disappointment, this attempt failed. The benzyl group was used to protect the hydroxyl groups. 1,3,4,6-Tetra$O$-benzyl- $\beta$-D-glucosamine hydrochloride 1 was synthesized according to the literature $[38,39]$. Treatment of compounds 1 with triethylamine in acetonitrile was followed by the addition of carbon disulfide, which furnished dithiocarbamic acid salt $\mathbf{2}$. Subsequent reaction of $\mathbf{2}$ with tosyl chloride ( $p$-TsCl) yielded the key glycosyl isothiocyanate immediate $\mathbf{3}$ (Scheme 1). Compound $\mathbf{3}$ was treated with various hydrazides $\mathbf{4}$ to yield the glycosyl acylthiosemicarbazide derivatives $\mathbf{5 a - j}$. Cyclization of the intermediate products 5 in a base-catalyzed reaction afforded the glycosyl oxadiazole derivatives $\mathbf{6 a - j}$ in high yields (Scheme 2).

\section{Biological activity}

The AChE-inhibition activities of the compounds were evaluated in vitro by Ellman's method [40] using the AChE extract from electric eel. The results are summarized in Table 1. Selected compounds were subsequently evaluated for the maximal inhibitory concentration, 


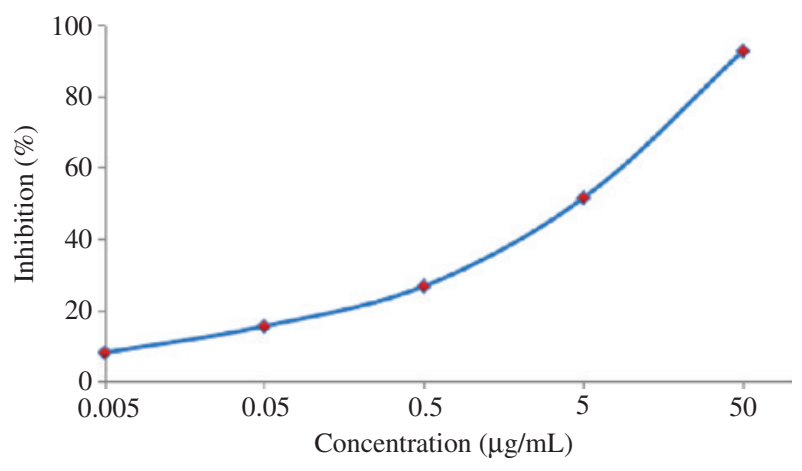

Figure 1 Dose-dependent inhibition of AChE by compound $\mathbf{6 i}$. Values are presented as mean $\pm S D, n=3$.

$\mathrm{IC}_{50}$, with tacrine and galantamine as the reference compounds. The results indicate that all compounds show higher inhibitory activities against AChE than the precursor compound $\mathbf{n}$. The best compound $\mathbf{6 i}$ shows the highest activity with an $\mathrm{IC}_{50}$ value of $1.61 \pm 0.34$ against AChE and inhibits AChE in a dose-dependent relationship (Figure 1). Compounds $\mathbf{1}$ and $\mathbf{m}$ demonstrate weak inhibition of AChE compared with compound $\mathbf{6 i}$, suggesting that the presence of 1,3,4-oxadiazole unit improves the activity.

\section{Conclusion}

A new series of C2-glycosyl oxadiazole derivatives were designed, synthesized and subjected to biological evaluation. These compounds were characterized by NMR, IR and HRMS. Most of the compounds are active against AChE. Compound $\mathbf{6}$ i shows the best AChE-inhibition activity with an $\mathrm{IC}_{50}$ of $1.61 \pm 0.34 \mu \mathrm{M}$.

\section{Experimental}

\section{Chemistry}

All chemicals were purchased from commercial sources and used without further purification. Melting points were determined on a Yanaco melting point apparatus and were uncorrected. IR spectra were recorded on a Bruker Tensor 27 spectrometer in $\mathrm{KBr}$ pellets. ${ }^{1} \mathrm{H}$ NMR spectra were recorded on a Bruker Avance $400 \mathrm{MHz}$ at ambient temperature using dimethyl sulfoxide- $d_{6}$ (DMSO- $d_{6}$ ) as a solvent and tetramethylsilane (TMS) as an internal standard. HRMS (ESI) analysis was performed on an Agilent 6230 mass spectrometer. Flash column chromatography was performed on silica 200-300 mesh.

\section{2-Amino-1,3,4,6-tetra- 0 -benzyl-2-deoxy- $\beta$-D- glucopyranose hydrochloride (1)}

A solution of D-glucosamine hydrochloride (10 g, $46.4 \mathrm{mmol}$ ) in water $(70 \mathrm{~mL})$ at room temperature was stirred and treated with $\mathrm{NaOH}$ (1.86 g, $46.5 \mathrm{mmol}$ ), and $15 \mathrm{~min}$ later dropwise with $p$-methoxybenzaldehyde $(5.7 \mathrm{~mL}, 46.6 \mathrm{mmol})$. The mixture was stirred at ambient temperature for an additional $24 \mathrm{~h}$, after which time the resulting white solid was filtered and washed with $500 \mathrm{~mL}$ of water to afford 2-(4-methoxy benzylidene)-2-deoxy- $\beta$-Dglucopyranose $(11.4 \mathrm{~g}, 83 \%)$. A mixture of this product and $\mathrm{BnBr}$ $(14 \mathrm{~mL}, 118 \mathrm{mmol})$ in dimethylformamide (DMF) $(50 \mathrm{~mL})$ at $0^{\circ} \mathrm{C}$ was treated portion-wise with $\mathrm{NaH}(60 \%, 5 \mathrm{~g}, 125 \mathrm{mmol})$. The mixture was stirred at room temperature for $12 \mathrm{~h}$, then diluted with a large amount of water and extracted with $\mathrm{CH}_{2} \mathrm{Cl}_{2}(3 \times 50 \mathrm{~mL})$. The extract was concentrated under reduced pressure to give a yellow viscous liquid. The solution of the yellow liquid in acetone $(100 \mathrm{~mL})$ was treated with hydrochloric acid $(7 \mathrm{~mL}, 5 \mathrm{~N})$ to afford a white solid of 1 after reflux for $1 \mathrm{~h}$. Product $\mathbf{1}$ was washed with acetone; yield $7.9 \mathrm{~g} \mathrm{(62 \% ).}$

\section{2-Isothiocyanato-1,3,4,6-tetra- $O$-benzyl-2-deoxy- $\beta$-D- glucopyranose (3)}

A solution of 1,3,4,6-tetra- $O$-benzyl- $\beta$-D-glucosamine hydrochloride $1(1 \mathrm{mmol})$ and triethylamine $(3 \mathrm{mmol})$ in acetonitrile $(15 \mathrm{~mL})$ was cooled in an ice bath and treated dropwise with carbon disulfide $(1 \mathrm{mmol})$. The mixture was stirred for $2 \mathrm{~h}$, then treated with $p$-TsCl $(1 \mathrm{mmol})$ and stirred for another $0.5 \mathrm{~h}$ on the ice bath. The precipitate of product 3 was crystallized from ethanol; yield $90 \%$ of white amorphous powder; $\mathrm{mp} 55-56^{\circ} \mathrm{C}$; IR: 3433, 3030, 2873, 2078, 1454 , 1359, 1313, $1068 \mathrm{~cm}^{-1}$; ${ }^{1} \mathrm{H}$ NMR: $\delta 7.45-7.25$ (m, 18H, ArH), 7.24-7.17 (dd, $J=7 \mathrm{~Hz}, 2 \mathrm{H}, \mathrm{ArH}), 4.81\left(\mathrm{dd}, J=16,10 \mathrm{~Hz}, 4 \mathrm{H}, \mathrm{PhCH}_{2}, \mathrm{HGlu}\right), 4.73-4.62$ (m, 2H, $\mathrm{PhCH}_{2}$ ), 4.61-4.48 (m, 3H, $\mathrm{PhCH}_{2}$ ), 3.95-3.87 (m, 2H, HGlu), 3.67 (ddd, $J=14,12,7 \mathrm{~Hz}, 3 \mathrm{H}, \mathrm{HGlu}$ ), 3.54 (dd, $J=12,7 \mathrm{~Hz}, 1 \mathrm{H}, \mathrm{HGlu}$ ); ESI-HRMS $(\mathrm{m} / z)$ : Calcd for $\mathrm{C}_{35} \mathrm{H}_{35} \mathrm{NNaO}_{5} \mathrm{~S}[\mathrm{M}+\mathrm{Na}]^{+}:$604.2128; found: 604.2130.

\section{General procedure for the preparation of $N$-(1,3,4,6-tetr- a-O-benzyl-2-deoxy- $\beta$-D-glucopyranosyl)-5-aryl-1,3,4- oxadiazole-2-amines $6 \mathrm{a}-\mathrm{j}$}

Glycosyl isothiocyanate $3(0.58 \mathrm{~g}, 1 \mathrm{mmol})$ was added in one portion to a stirred solution of hydrazide $4(1 \mathrm{mmol})$ in $\mathrm{MeCN}(10 \mathrm{~mL})$. The reaction mixture was heated under reflux for $3-4 \mathrm{~h}$, and then the solvent was eliminated under reduced pressure. The residue was crystallized from aqueous ethanol to obtain the desired product $\mathbf{5 a}-\mathbf{j} \cdot p$ - $\mathrm{TsCl}(0.21 \mathrm{~g}, 1.1 \mathrm{mmol})$ was added to a stirred solution of acylthiosemicarbazide $5(1 \mathrm{mmol})$ and triethylamine $(0.16 \mathrm{~mL}$, $2.0 \mathrm{mmol})$ in MeCN $(10 \mathrm{~mL})$. The mixture was stirred for about $2-3 \mathrm{~h}$ at $81^{\circ} \mathrm{C}$, and the reaction progress was monitored by thinlayer chromatography (TLC). The desired compound $\mathbf{6 a - j}$ was purified by column chromatography on silica gel eluting with ethyl acetate/petroleum ether (1:2) to give a white amorphous product. 
$N$-(1,3,4,6-tetra- $O$-benzyl-2-deoxy- $\beta$-D-glucopyranosyl)-5phenyl-1,3,4-oxadiazole-2-amine (6a) Yield $86 \%$; mp $166-168^{\circ} \mathrm{C}$; IR (cm-1): 3421, 3226, 3060, 2924, 1630, 1496, 1453, 1397, 1116, 1064; ${ }^{1} \mathrm{H}$ NMR: $\delta 8.15$ (d, $\left.J=9 \mathrm{~Hz}, 1 \mathrm{H}, \mathrm{NH}\right), 7.86-7.80$ (m, 2H, ArH), 7.56-7.50 (m, 3H, ArH), 7.40-7.27 (m, 8H, ArH), 7.25-7.12 (m, 12H, ArH), 4.82 (d, $\left.J=12 \mathrm{~Hz}, 1 \mathrm{H}, \mathrm{H}-1^{\mathrm{Glu}}\right), 4.80-4.65\left(\mathrm{~m}, 4 \mathrm{H}, \mathrm{PhCH}_{2}\right), 4.62-4.51$ (m, 4H, $\mathrm{PhCH}_{2}$ ), 3.85-3.69 (m, 3H, H-4 $\left.{ }^{\mathrm{Glu}}, \mathrm{H}-6 \mathrm{a}^{\mathrm{Glu}}, \mathrm{H}-6 \mathrm{~b}^{\mathrm{Glu}}\right)$, 3.65-3.50 (m, $3 \mathrm{H}, \mathrm{H}-5^{\mathrm{Glu}}$, H-3 ${ }^{\mathrm{Glu}}$, H-2 ${ }^{\mathrm{Glu}}$ ); ESI-HRMS $(\mathrm{m} / z)$ : Calcd for $\mathrm{C}_{42} \mathrm{H}_{41} \mathrm{~N}_{3} \mathrm{NaO}_{6}$ $[\mathrm{M}+\mathrm{Na}]^{+}:$706.2888; found: 706.2890 .

$\boldsymbol{N}$-(1,3,4,6-tetra- $\boldsymbol{O}$-benzyl-2-deoxy- $\boldsymbol{\beta}$-D-glucopyranosyl)-5-(4methylphenyl)-1,3,4-oxadiazole-2-amine (6b) Yield 88\%; mp 159-160 ${ }^{\circ} \mathrm{C}$; IR $\left(\mathrm{cm}^{-1}\right): 3446,3170,2923,1629,1400,1071,1028 ;{ }^{1} \mathrm{H}$ NMR: $\delta 8.08$ (d, $J=9 \mathrm{~Hz}, 1 \mathrm{H}, \mathrm{NH}), 7.71$ (d, $J=8 \mathrm{~Hz}, 2 \mathrm{H}, \mathrm{ArH}), 7.42-7.27$ (m, 10H, ArH), 7.25-7.13 (m, 12H, ArH), 4.82 (d, J=12 Hz, 1H, H-1 ${ }^{\mathrm{Glu}}$ ), 4.78-4.70 (m, 3H, $\mathrm{PhCH}_{2}$ ), 4.68-4.52 (m, 5H, $\mathrm{PhCH}_{2}$ ), 3.83-3.68 (m, 3H, $\left.\mathrm{H}-4^{\mathrm{Glu}}, \mathrm{H}-6 \mathrm{a}^{\mathrm{Glu}}, \mathrm{H}-6 \mathrm{~b}^{\mathrm{Glu}}\right), 3.63-3.52$ (m, 3H, H-5 $\left.{ }^{\mathrm{Glu}}, \mathrm{H}-3^{\mathrm{Glu}}, \mathrm{H}-2^{\mathrm{Glu}}\right), 2.40-$ $2.32\left(\mathrm{~s}, 3 \mathrm{H}, \mathrm{CH}_{3}\right)$; ESI-HRMS $(\mathrm{m} / \mathrm{z})$ : Calcd for $\mathrm{C}_{43} \mathrm{H}_{43} \mathrm{~N}_{3} \mathrm{NaO}_{6}[\mathrm{M}+\mathrm{Na}]^{+}$: 720.3044; found: 720.3046 .

$\boldsymbol{N}$-(1,3,4,6-tetra- 0 -benzyl-2-deoxy- $\boldsymbol{\beta}$-D-glucopyranosyl)-5-(2thienyl)-1,3,4-oxadiazole-2-amine (6c) Yield 84\%; mp 147-148 ${ }^{\circ} \mathrm{C}$; IR $\left(\mathrm{cm}^{-1}\right)$ : 3420, 3201, 3026, 2923, 1496, 1468, 1400, 1114, 1066; ${ }^{1} \mathrm{H}$ NMR: $\delta 8.36$ (d, $J=9 \mathrm{~Hz}, 1 \mathrm{H}, \mathrm{NH}), 7.66(\mathrm{~d}, J=8 \mathrm{~Hz}, 1 \mathrm{H}, \mathrm{ArH}), 7.42$ (d, $J=4 \mathrm{~Hz}, 1 \mathrm{H}, \mathrm{ArH}$ ), 7.40-7.29 (m, 8H, ArH), 7.27-7.11 (m, 13H, ArH), 4.82 (d, J=12.5 Hz, 1H, H-1 ${ }^{\mathrm{Glu}}$ ), 4.76-4.65 (m, 4H, $\mathrm{PhCH}_{2}$ ), 4.63-4.52 (m, 4H, $\mathrm{PhCH}_{2}$ ), 3.86 (t, J=8 Hz, 1H, H-4 ${ }^{\mathrm{Glu}}$ ), 3.78-3.67 (m, 2H, H-6a ${ }^{\mathrm{Glu}}, \mathrm{H}-6 \mathrm{~b}^{\mathrm{Glu}}$ ), 3.61-3.50 (m, 3H, H-5 $\left.{ }^{\text {Glu }}, \mathrm{H}-3^{\mathrm{Glu}}, \mathrm{H}-2^{\mathrm{Glu}}\right)$; ESI-HRMS $(\mathrm{m} / \mathrm{z})$ : Calcd for $\mathrm{C}_{40} \mathrm{H}_{39} \mathrm{~N}_{3} \mathrm{NaO}_{6} \mathrm{~S}[\mathrm{M}+\mathrm{Na}]^{+}:$712.2452; found: 712.2456.

$\boldsymbol{N}$-(1,3,4,6-tetra- $\boldsymbol{O}$-benzyl-2-deoxy- $\boldsymbol{\beta}$-D-glucopyranosyl)-5-(4pyridyl)-1,3,4-oxadiazole-2-amine (6d) Yield $86 \%$; mp $182-183^{\circ} \mathrm{C}$; IR ( $\left.\mathrm{cm}^{-1}\right)$ : 3421, 3230, 3060, 2924, 1627, 1465, 1398, 1061, 1028; ${ }^{1} \mathrm{H}$ NMR: $\delta$ 8.98 (d, $J=2 \mathrm{~Hz}, 1 \mathrm{H}, \mathrm{ArH}), 8.70$ (dd, $J=5,2 \mathrm{~Hz}, 1 \mathrm{H}, \mathrm{ArH}), 8.26$ (d, $J=9 \mathrm{~Hz}$, $1 \mathrm{H}, \mathrm{NH}), 8.16$ (2t, $J=2 \mathrm{~Hz}, 1 \mathrm{H}, \mathrm{ArH}), 7.56$ (dd, $J=8.5 \mathrm{~Hz}, 1 \mathrm{H}, \mathrm{ArH}), 7.40-$ 7.27 (m, 8H, ArH), 7.25-7.10 (m, 12H, ArH), 4.82 (d, J=12 Hz, 1H, H-1 ${ }^{\mathrm{Glu}}$ ), 4.79-4.64 (m, 4H, $\mathrm{PhCH}_{2}$ ), 4.62-4.52 (m, 4H, $\left.\mathrm{PhCH}_{2}\right), 3.84-3.69$ (m, 3H, $\left.\mathrm{H}-4^{\mathrm{Glu}}, \mathrm{H}-6 \mathrm{a}^{\mathrm{Glu}}, \mathrm{H}-6 \mathrm{~b}^{\mathrm{Glu}}\right), 3.65-3.55$ (m, 3H, H-5 $\left.5^{\mathrm{Glu}}, \mathrm{H}-3^{\mathrm{Glu}}, \mathrm{H}-2^{\mathrm{Glu}}\right)$; ESI-HRMS $(\mathrm{m} / z)$ : Calcd for $\mathrm{C}_{41} \mathrm{H}_{40} \mathrm{~N}_{4} \mathrm{NaO}_{6}[\mathrm{M}+\mathrm{Na}]^{+}$: 707.2840; found: 707.2843.

$\boldsymbol{N}$-(1,3,4,6-tetra- $\boldsymbol{O}$-benzyl-2-deoxy- $\boldsymbol{\beta}$-D-glucopyranosyl)-5-(3methoxyphenyl)-1,3,4-oxadiazole-2-amine (6e) Yield 90\%; mp $147-148^{\circ} \mathrm{C}$; IR $\left(\mathrm{cm}^{-1}\right)$ : 3426, 3174, 3028, 2926, 1598, 1497, 1453, 1400, 1216, 1125, 1069, 1043; ${ }^{1} \mathrm{H}$ NMR: $(\delta 8.37(\mathrm{~d}, J=9 \mathrm{~Hz}, 1 \mathrm{H}, \mathrm{NH}), 7.43-7.27$ (m, 12H, ArH), 7.25-7.15 (m, 11H, ArH), 7.05-7.01 (m, 1H, ArH), 4.83 (d, $\left.J=12.5 \mathrm{~Hz}, 1 \mathrm{H}, \mathrm{H}-1^{\mathrm{Glu}}\right), 4.78-4.67$ (m, 4H, $\left.\mathrm{PhCH}_{2}\right), 4.64-4.51$ (m, 4H, $\mathrm{PhCH}_{2}$ ), 3.87 (t, $\left.J=8 \mathrm{~Hz}, 1 \mathrm{H}, \mathrm{H}-4^{\mathrm{Glu}}\right), 3.82\left(\mathrm{~s}, 3 \mathrm{H}, \mathrm{OCH}_{3}\right), 3.77-3.68(\mathrm{~m}$, 2H, H-6a ${ }^{\mathrm{Glu}}, \mathrm{H}-6 \mathrm{~b}^{\mathrm{Glu}}$ ), 3.61-3.51 (m, 3H, H-5 $5^{\mathrm{Glu}}, \mathrm{H}-3^{\mathrm{Glu}}$, H-2 ${ }^{\mathrm{Glu}}$ ); ESI-HRMS $(\mathrm{m} / z)$ : Calcd for $\mathrm{C}_{43} \mathrm{H}_{43} \mathrm{~N}_{3} \mathrm{NaO}_{7}[\mathrm{M}+\mathrm{Na}]^{+}:$736.2993; found: 736.2998.

$\boldsymbol{N}$-(1,3,4,6-tetra- $\boldsymbol{O}$-benzyl-2-deoxy- $\boldsymbol{\beta}$-D-glucopyranosyl)-5-(2chlorophenyl)-1,3,4-oxadiazole-2-amine (6f) Yield 83\%; mp 121$122^{\circ} \mathrm{C}$; IR $\left(\mathrm{cm}^{-1}\right)$ : 3421, 3231, 3062, 2924, 1627, 1508, 1454, 1397, 1362, 1149, 1076, 1028; ${ }^{1} \mathrm{H}$ NMR: $\delta 8.25$ (d, $\left.J=9 \mathrm{~Hz}, 1 \mathrm{H}, \mathrm{NH}\right), 7.78$ (dd, $J=8$, $1.8 \mathrm{~Hz}, 1 \mathrm{H}, \mathrm{ArH}), 7.65$ (dd, $J=8,1.1 \mathrm{~Hz}, 1 \mathrm{H}, \mathrm{ArH}), 7.58-7.47$ (m, 2H, ArH), 7.40-7.27 (m, 8H, ArH), 7.26-7.13 (m, 12H, ArH), 4.83 (d, J=12.5 Hz, 1H, $\mathrm{H}-\mathrm{-}^{\mathrm{Glu}}$ ), 4.79-4.63 (m, 4H, $\mathrm{PhCH}_{2}$ ), 4.62-4.51 (m, 4H, $\left.\mathrm{PhCH}_{2}\right), 3.84-3.68$ (m, 3H, H-4 ${ }^{\mathrm{Glu}}, \mathrm{H}-6 \mathrm{a}^{\mathrm{Glu}}, \mathrm{H}-6 \mathrm{~b}^{\mathrm{Glu}}$ ), 3.63-3.50 (m, 3H, H-5 $5^{\mathrm{Glu}}, \mathrm{H}-3^{\mathrm{Glu}}, \mathrm{H}-2^{\mathrm{Glu}}$ ); ESI-HRMS $(\mathrm{m} / z)$ : Calcd for $\mathrm{C}_{42} \mathrm{H}_{40} \mathrm{ClN}_{3} \mathrm{NaO}_{6}[\mathrm{M}+\mathrm{Na}]^{+}: 740.2498$; found: 740.2501 .
$N$-(1,3,4,6-tetra-O-benzyl-2-deoxy- $\beta$-D-glucopyranosyl)-5-(4hydroxylphenyl)-1,3,4-oxadiazole-2-amine (6g) Yield 86\%; mp 181-182 ${ }^{\circ}$; IR ( $\left.\mathrm{cm}^{-1}\right)$ : 3421, 3142, 3030, 2956, 1650, 1611, 1497, 1398, 1279, 1173, 1072, 1027; ${ }^{1} \mathrm{H}$ NMR: $\delta 10.10$ (s, $\left.1 \mathrm{H}, \mathrm{OH}\right), 7.98$ (d, $J=9 \mathrm{~Hz}, 1 \mathrm{H}$, $\mathrm{NH}), 7.66$ (t, $J=9 \mathrm{~Hz}, 2 \mathrm{H}, \mathrm{ArH}), 7.41-7.12(\mathrm{~m}, 20 \mathrm{H}, \mathrm{ArH}), 6.89$ (d, $J=9 \mathrm{~Hz}$, $2 \mathrm{H}, \mathrm{ArH}), 4.81$ (d, $\left.J=12 \mathrm{~Hz}, 1 \mathrm{H}, \mathrm{H}-1^{\mathrm{Glu}}\right), 4.78-4.50$ (m, 8H, $\mathrm{PhCH}_{2}$ ), 3.833.68 (m, 3H, H-4 $\left.4^{\mathrm{Glu}}, \mathrm{H}-6 \mathrm{a}^{\mathrm{Glu}}, \mathrm{H}-6 \mathrm{~b}^{\mathrm{Glu}}\right)$, 3.69-3.50 (m, 3H, H-5 $5^{\mathrm{Glu}}, \mathrm{H}-3^{\mathrm{Glu}}$, $\left.\mathrm{H}-2^{\mathrm{Glu}}\right)$; ESI-HRMS $(\mathrm{m} / z)$ : Calcd for $\mathrm{C}_{42} \mathrm{H}_{41} \mathrm{~N}_{3} \mathrm{NaO}_{7}[\mathrm{M}+\mathrm{Na}]^{+}$: 722.2837; found: 722.2838 .

$\boldsymbol{N}$-(1,3,4,6-tetra-O-benzyl-2-deoxy- $\boldsymbol{\beta}$-D-glucopyranosyl)-5-(4iodinylphenyl)-1,3,4-oxadiazole-2-amine (6h) Yield 82\%; mp 176-177 ${ }^{\circ}$; IR ( $\left.\mathrm{cm}^{-1}\right)$ : 3446, 3229, 3059, 2922, 1627, 1478, 1453, 1397, 1362, 1203, 1055, 1005; ${ }^{1} \mathrm{H}$ NMR: $\delta 8.19$ (d, J=9 Hz, 1H, NH), 7.90 (d, $J=8.5 \mathrm{~Hz}, 2 \mathrm{H}, \mathrm{ArH}), 7.58$ (d, $J=8.5 \mathrm{~Hz}, 2 \mathrm{H}, \mathrm{ArH}), 7.40-7.27$ (m, 8H, ArH), 7.25-7.10 (m, 12H, ArH), 4.81 (d, $\left.J=12 \mathrm{~Hz}, 1 \mathrm{H}, \mathrm{H}-1^{\mathrm{Glu}}\right), 4.77-4.61$ (m, 4H, $\mathrm{PhCH}_{2}$ ), 4.60-4.50 (m, 4H, $\mathrm{PhCH}_{2}$ ), 3.83-3.67 (m, 3H, H-4 ${ }^{\mathrm{Glu}}$, H-6a ${ }^{\mathrm{Glu}}, \mathrm{H}-6 \mathrm{~b}^{\mathrm{Glu}}$ ), 3.64-3.51 (m, 3H, H-5 $5^{\mathrm{Glu}}, \mathrm{H}-3^{\mathrm{Glu}}, \mathrm{H}-2^{\mathrm{Glu}}$ ); ESI-HRMS $(\mathrm{m} / z)$ : Calcd for $\mathrm{C}_{42} \mathrm{H}_{40} \mathrm{IN}_{3} \mathrm{NaO}_{6}[\mathrm{M}+\mathrm{Na}]^{+}$: 832.1854; found: 832.1860.

$\boldsymbol{N}$-(1,3,4,6-tetra- $\boldsymbol{O}$-benzyl-2-deoxy- $\boldsymbol{\beta}$-D-glucopyranosyl)-5-(4fluorophenyl)-1,3,4-oxadiazole-2-amine (6i) Yield 88\%; mp 156$157^{\circ} \mathrm{C}$; IR $\left(\mathrm{cm}^{-1}\right)$ : 3425, 3179, 3029, 2923, 1601, 1518, 1498, 1400, 1219, 1124, 1070; ${ }^{1} \mathrm{H}$ NMR: $\delta 8.34$ (d, $\left.J=9 \mathrm{~Hz}, 1 \mathrm{H}, \mathrm{NH}\right), 7.84-7.77$ (m, 2H, ArH), 7.41-7.26 (m, 11H, ArH), 7.25-7.13 (m, 11H, ArH), 4.82 (d, J=12.5 Hz, $1 \mathrm{H}$, $\left.\mathrm{H}-1^{\mathrm{Glu}}\right)$, 4.76-4.66 (m, 4H, $\left.\mathrm{PhCH}_{2}\right), 4.63-4.51\left(\mathrm{~m}, 4 \mathrm{H}, \mathrm{PhCH}_{2}\right), 3.86(\mathrm{t}$, $\left.J=8 \mathrm{~Hz}, 1 \mathrm{H}, \mathrm{H}-4^{\mathrm{Glu}}\right), 3.78-3.67$ (m, 2H, H-6a $\left.{ }^{\mathrm{Glu}}, \mathrm{H}-6 \mathrm{~b}^{\mathrm{Glu}}\right), 3.62-3.52$ (m, $\left.3 \mathrm{H}, \mathrm{H}-5^{\mathrm{Glu}}, \mathrm{H}-3^{\mathrm{Glu}}, \mathrm{H}-2^{\mathrm{Glu}}\right)$; ESI-HRMS $(\mathrm{m} / z)$ : Calcd for $\mathrm{C}_{42} \mathrm{H}_{40} \mathrm{FN}_{3} \mathrm{NaO}_{6}$ $[\mathrm{M}+\mathrm{Na}]^{+}$: 724.2793; found: 724.2796 .

$N$-(1,3,4,6-tetra-O-benzyl-2-deoxy- $\beta$-D-glucopyranosyl)-5-(4dimethylaminophenyl)-1,3,4-oxadiazole-2-amine (6j) Yield 83\%; mp 123-124 ${ }^{\circ} \mathrm{C}$; IR $\left(\mathrm{cm}^{-1}\right)$ : 3422, 3234, 3060, 2906, 1633, 1614, 1519, 1397, 1197, 1068, 1028; ${ }^{1} \mathrm{H}$ NMR: $\delta 7.89$ (d, $\left.J=9 \mathrm{~Hz}, 1 \mathrm{H}, \mathrm{NH}\right), 7.62$ (d, J=9 Hz, 2H, ArH), 7.41-7.25 (m, 8H, ArH), 7.24-7.15 (m, 12H, ArH), 6.80 (d, $J=9 \mathrm{~Hz}, 2 \mathrm{H}, \mathrm{ArH}), 4.81$ (d, $J=12.5 \mathrm{~Hz}, 1 \mathrm{H}, \mathrm{H}-\mathbf{-}^{\mathrm{Glu}}$ ), 4.78-4.62 (m, 4H, $\mathrm{PhCH}_{2}$ ), 4.60-4.51 (m, 4H, $\mathrm{PhCH}_{2}$ ), 3.83-3.66 (m, 3H, H-4 $4^{\mathrm{Glu}}$, H-6a ${ }^{\mathrm{Glu}}, \mathrm{H}-6 \mathrm{~b}^{\mathrm{Glu}}$ ), 3.63-3.49 (m, 3H, H-5 $5^{\mathrm{Glu}}, \mathrm{H}-3^{\mathrm{Glu}}, \mathrm{H}-2^{\mathrm{Glu}}$ ), 2.98 (s, 6H, $\mathrm{CH}_{3}$ ); ESI-HRMS $(\mathrm{m} / z)$ : Calcd for $\mathrm{C}_{44} \mathrm{H}_{46} \mathrm{~N}_{4} \mathrm{NaO}_{6}[\mathrm{M}+\mathrm{Na}]^{+}$: 749.3310; found: 749.3312 .

\section{In vitro cholinesterase activity assay}

AChE, acetylthiocholine iodide (ATCI), 5,5-dithiobis-(2-nitrobenzoic acid) (DTNB), galantamine and tacrine were purchased from SigmaAldrich. AChE activities were measured using Ellman's colorimetric method with a slight modification [39]; galantamine and tacrine were the reference compounds. For the determination, a 96-well plate was used as the carrier. First, $130 \mu \mathrm{L}$ of buffer solution, $20 \mu \mathrm{L}$ of AChE solution, $20 \mu \mathrm{L}$ of color developer and $10 \mu \mathrm{L}$ of methanol were added to the first column of the 96 -well plate as a control blank system. Then, $130 \mu \mathrm{L}$ of buffer solution, $20 \mu \mathrm{L}$ of AChE solution, $20 \mu \mathrm{L}$ of developer and $10 \mu \mathrm{L}$ of the analyte solution were added. After all samples were added, the plate was treated with $20 \mu \mathrm{L}$ of substrate and shaken evenly. The plate was quickly placed in the microplate reader and the temperature was maintained at $20-25^{\circ} \mathrm{C}$. The reaction rates were compared and the percent inhibition due to the presence of tested compounds was calculated. All samples were assayed in triplicate. 
The $50 \%$ inhibitory concentration $\left(\mathrm{IC}_{50}\right)$ was calculated from a doseresponse curve obtained by plotting the percentage of inhibition versus the log concentration with the use of the Origin 8.0 software. The results were described as mean \pm standard deviation (SD).

Acknowledgments: This work was supported by the Postgraduate Research and Practice Innovation Program of Jiangsu Province (KYCX17-2074), Natural Science Foundation of Jiangsu Province (BK20151281), Open-end Funds of Jiangsu Key Laboratory of Marine Biotechnology (HS2014007) and Public Science and Technology Research Funds Projects of Ocean (201505023).

\section{References}

[1] Tsvetkov, Y. E.; Burg-Roderfeld, M.; Loers, G.; Ardá, A.; Sukhova, E. V.; Khatuntseva, E. A.; Jiménez-Barbero, J. Synthesis and molecular recognition studies of the HNK-1 trisaccharide and related oligosaccharides. The specificity of monoclonal anti-HNK-1 antibodies as assessed by surface plasmon resonance and STD NMR. J. Am. Chem. Soc. 2011, 134, 426-435.

[2] Kimura, M.; Masui, Y.; Shirai, Y.; Honda, C.; Moriwaki, K.; Imai, T.; Nakano, H. Preparation of branched cyclomaltoheptaose with 3- $O$ - $\alpha$-L-fucopyranosyl- $\alpha$-D-mannopyranose and changes in fucosylation of HCT116 cells treated with the fucose-modified cyclomaltoheptaose. Carbohydr. Res. 2013, 374, 49-58.

[3] Delbianco, M.; Bharate, P.; Varela-Aramburu, S.; Seeberger, P. H. Carbohydrates in supramolecular chemistry. Chem. Rev. 2016, 116, 1693-1752.

[4] Ernst, B.; Magnani, J. L. From carbohydrate leads to glycomimetic drugs. Nat. Rev. Drug Discov. 2009, 8, 661-677.

[5] Bertozzi, C. R.; Kiessling, L. L. Chemical glycobiology. Science 2001, 291, 2357-2364.

[6] Xie, J. Synthesis of new sugar amino acid derivatives of D-glucosamine. Carbohydr. Res. 2003, 338, 399-405.

[7] Xu, C.; Hall, R.; Cummings, J.; Raushel, F. M. Tight binding inhibitors of $\mathrm{N}$-acyl amino sugar and $\mathrm{N}$-acyl amino acid deacetylases. J. Am. Chem. Soc. 2006, 128, 4244-4245.

[8] Fu, M.; Qin, C.; Li, W.; Yan, Y.; Zeng, L.; Yang, X. Effect of glucosamine and chitooligomer on the toxicity of arsenite against Escherichia coli. Carbohyd. Polym. 2013, 91, 390-396.

[9] Terencio, M. C.; Ferrándiz, M. L.; Carceller, M. C.; Ruhí, R.; Dalmau, P.; Vergés, J.; Alcaraz, M. J. Chondroprotective effects of the combination chondroitin sulfate-glucosamine in a model of osteoarthritis induced by anterior cruciate ligament transection in ovariectomised rats. Biomed. Pharmacother. 2016, 79, 120-128.

[10] Nagaoka, I.; Igarashi, M.; Hua, J.; Ju, Y.; Yomogida, S.; Sakamoto, K. Recent aspects of the anti-inflammatory actions of glucosamine. Carbohyd. Polym. 2011, 84, 825-831.

[11] Oh, H. J.; Lee, J. S.; Song, D. K.; Shin, D. H.; Jang, B. C.; Suh, S. I.; Baek, W. K. D-glucosamine inhibits proliferation of human cancer cells through inhibition of p70S6K. Biochem. Bioph. Res. Commun. 2007, 360, 840-846.
[12] Blagodatskikh, I. V.; Kulikov, S. N.; Vyshivannaya, O. V.; Bezrodnykh, E. A.; Yamskov, I. A.; Tikhonov, V. E. Influence of glucosamine on oligochitosan solubility and antibacterial activity. Carbohydr. Res. 2013, 381, 28-32.

[13] Quastel, J. Inhibition of tumour growth by D-glucosamine. Nature 1953, 171, 252-254.

[14] Xing, R.; Liu, S.; Guo, Z.; Yu, H.; Li, C.; Ji, X.; Feng, J.; Li, P. The antioxidant activity of glucosamine hydrochloride in vitro. Bioorg. Med. Chem. 2006, 14, 1706-1709.

[15] Liu, W. W.; Li, Q. X.; Shi, D. H. Synthesis, characterization, and biological evaluation of some novel glycosyl 1,3,4-thiadiazole derivatives as acetylcholinesterase inhibitors. Heterocycles 2015, 91, 275-286.

[16] Punganuru, S. R.; Mostofa, A. G. M.; Madala, H. R.; Basak, D.; Srivenugopal, K. S. Potent anti-proliferative actions of a non-diuretic glucosamine derivative of ethacrynic acid. Bioorg. Med. Chem. Lett. 2016, 26, 2829-2835.

[17] Garifullin, B. F.; Strobykina, I. Y.; Sharipova, R. R.; Kravchenko, M. A.; Andreeva, O. V.; Bazanova, O. B.; Kataev, V. E. Synthesis and antituberculosis activity of the first macrocyclic glycoterpenoids comprising glucosamine and diterpenoid isosteviol. Carbohydr. Res. 2016, 431, 15-21.

[18] Yang, J.; Li, J. R.; Yang, J. X.; Li, L. L.; Ouyang, W. J.; Wu, S. W.; Zhang, F. Synthesis and anti-HIV-1 activity of the conjugates of gossypol with oligopeptides and D-glucosamine. Chinese Chem. Lett. 2014, 25, 1052-1058.

[19] Mendis, E.; Kim, M. M.; Rajapakse, N.; Kim, S. K. The inhibitory mechanism of a novel cationic glucosamine derivative against MMP-2 and MMP-9 expressions. Bioorg. Med. Chem. Lett. 2009, 19, 2755-2760.

[20] Shen, C.; Shen, H.; Yang, M.; Xia, C.; Zhang, P. A novel D-glucosamine-derived pyridyl-triazole for solvent-free Mizoroki-Heck reactions and its application in the synthesis of Axitinib. Green Chem. 2015, 17, 225-230.

[21] Bepary, S.; Yoon, I. K.; Lee, G. H. Novel 3-amino7-(aminomethyl)-1H-indazol-4-ol as the PI3K $\gamma$ enzyme inhibitor. Bull. Korean. Chem. Soc. 2016, 37, 2054-2057.

[22] Dighe, S. U.; Khan, S.; Soni, I.; Jain, P.; Shukla, S.; Yadav, R.; Sen, P.; Meeran, S. M.; Batra, S. Synthesis of $\beta$-carboline-based $\mathrm{N}$-heterocyclic carbenes and their antiproliferative and antimetastatic activities against human breast cancer cells. J. Med. Chem. 2015, 58, 3485-3499.

[23] Chen, Y.; Yu, K.; Tan, N. Y.; Qiu, R. H.; Liu, W.; Luo, N. L.; Yin, S. F. Synthesis, characterization and anti-proliferative activity of heterocyclic hypervalent organoantimony compounds. Eur. J. Med. Chem. 2014, 79, 391-398.

[24] Mathew, V.; Keshavayya, J.; Vaidya, V. P. Heterocyclic system containing bridgehead nitrogen atom: synthesis and pharmacological activities of some substituted 1,2,4-triazolo [3,4-b]-1,3,4thiadiazoles. Eur. J. Med. Chem. 2006, 41, 1048-1055.

[25] Borg, S.; Estenne-Bouhtou, G.; Luthman, K.; Csoeregh, I.; Hesselink, W.; Hacksell, U. Synthesis of 1,2,4-oxadiazole-, 1,3,4-oxadiazole- and 1,2,4-triazole-derived dipeptidomimetics. J. Org. Chem. 1995, 60, 3112-3117.

[26] Li, P.; Yin, J.; Xu, W.; Wu, J.; He, M.; Hu, D.; Song, B. Synthesis, antibacterial activities, and 3D-QSAR of sulfone derivatives containing 1,3,4-oxadiazole moiety. Chem. Biol. Drug Des. 2013, 82, 546-551.

[27] Padmaja, A.; Pedamalakondaiah, D.; Sravya, G.; Reddy, G. M.; Kumar, M. V. J. Synthesis and antioxidant activity of a 
new class of sulfone/sulfonamide-linked bis(oxadiazoles), bis(thiadiazoles), and bis(triazoles). J. Med. Chem. Res. 2015, 24, 2011-2020.

[28] Wu, W.; Chen, Q.; Tai, A.; Jiang, G.; Ouyang, G. Synthesis and antiviral activity of 2-substituted methylthio-5-(4-amino2-methylpyrimidin-5-yl)-1,3,4-oxadiazole derivatives. Bioorg. Med. Chem. Lett. 2015, 25, 2243-2250.

[29] Zhang, M. Z.; Mulholland, N.; Beattie, D.; Irwin, D.; Gu, Y. C.; Chen, Q.; Clough, J. Synthesis and antifungal activity of 3-(1,3,4-oxadiazol-5-yl)indoles and 3-(1,3,4-oxadiazol-5-yl) methylindoles. Eur. J. Med. Chem. 2013, 63, 22-28.

[30] Dekhane, D. V.; Pawar, S. S.; Gupta, S.; Shingare, M. S.; Patil, C. R.; Thore, S. N. Synthesis and anti-inflammatory activity of some new 4,5-dihydro-1,5-diaryl-1H-pyrazole-3-substituted heteroazole derivatives. Bioorg. Med. Chem. Lett. 2011, 21, 6527-6531.

[31] Zhang, Y.; Liu, X. H.; Zhan, Y. Z.; Zhang, L. Y.; Li, Z. M.; Li, Y. H.; Wang, B. L. Synthesis and biological activities of novel 5-substituted-1,3,4-oxadiazole Mannich bases and bis-Mannich bases as ketol-acid reductoisomerase inhibitors. Bioorg. Med. Chem. Lett. 2016, 26, 4661-4667.

[32] Distinto, S.; Meleddu, R.; Yanez, M.; Cirilli, R.; Bianco, G.; Sanna, M. L.; Ortuso, F. Drug design, synthesis, in vitro and in silico evaluation of selective monoaminoxidase $B$ inhibitors based on 3-acetyl-2-dichlorophenyl-5-aryl-2,3-dihydro-1,3,4-oxadiazole chemical scaffold. Eur. J. Med. Chem. 2016, 108, 542-548.

[33] Ke, S.; Li, Z.; Qian, X. 1,3,4-Oxadiazole-3(2H)-carboxamide derivatives as potential novel class of monoamine oxidase
(MAO) inhibitors: synthesis, evaluation, and role of urea moiety. Bioorg. Med. Chem. 2008, 16, 7565-7572.

[34] Mazouz, F.; Lebreton, L.; Milcent, R.; Burstein, C. 5-Aryl-1,3,4oxadiazol-2 (3H)-one derivatives and sulfur analogues as new selective and competitive monoamine oxidase type $B$ inhibitors. Eur. J. Med. Chem. 1990, 25, 659-663.

[35] Raza, R.; Saeed, A.; Arif, M.; Mahmood, S.; Muddassar, M.; Raza, A.; Iqbal, J. Synthesis and biological evaluation of 3-thiazolocoumarinyl Schiff-base derivatives as cholinesterase inhibitors. Chem. Biol. Drug Des. 2012, 80, 605-615.

[36] Wei, H.; Ruan, J.; Zhang, X. Coumarin-chalcone hybrids: promising agents with diverse pharmacological properties. RSC Adv. 2016, 6, 10846-10851.

[37] Havrylyuk, D.; Roman, O.; Lesyk, R. Synthetic approaches, structure activity relationship and biological applications for pharmacologically attractive pyrazole/pyrazoline-thiazolidinebased hybrids. Eur. J. Med. Chem. 2016, 113, 145-150.

[38] Cheng, F. C.; Yin, L.; Liu, W. W.; Li, Q. X.; Tang, L. J.; Shi, D. H.; Cao, Z. L. Expedient synthesis of novel glycosyl thiazole derivatives. Heterocycles 2015, 91, 2113-2125.

[39] Tao, C. Z.; Liu, F.; Liu, W. W.; Zhu, Y. M.; Li, Y. F.; Liu, X. L.; Zhao, J. Synthesis of $\mathrm{N}$-aryl-D-glucosamines through copper-catalyzed C-N coupling. Tetrahedron Lett. 2012, 53, 7093-7096.

[40] Ellman, G. L.; Courtney, K. D.; Andres Jr, V.; Featherstone, R. M. A new and rapid colorimetric determination of acetylcholinesterase activity. Biochem. Pharmacol. 1961, 7, 88-95. 